\title{
Influence of IL-6 and TNF- $a$ on clinical manifestations, activity and comorbidity in a group of patients with psoriatic arthritis
}

Carlos Montilla ( $\sim$ montillamorales.carlos@gmail.com )

Hospital Clínico Universitario Salamanca https://orcid.org/0000-0003-2258-2868

Gómez-Lechón Luis

Hospital Clinico de Salamanca

Esther Toledano

Hospital Clinico Universitario San Carlos

Elisa Acosta

Hospital Universitario de Salamanca

Olga Compán

Hospital Universitario de Salamanca

Sonia Pastor

Hospital Clinico de Salamanca

Cristina Hidalgo

Hospital Clinico de Salamanca

Guadalupe Manzano

Hospital Clinico de Salamanca

Rubén Queiró

Hospital Universitario Central de Asturias

Research article

Keywords: IL-6, TNF-a, comorbidities, psoriatic arthritis

Posted Date: August 2nd, 2020

DOI: https://doi.org/10.21203/rs.3.rs-49003/v1

License: (c) (i) This work is licensed under a Creative Commons Attribution 4.0 International License.

Read Full License 


\section{Abstract \\ Objectives}

To relate the levels of IL-6 and TNF-a in patients with psoriatic arthritis (PsA) with the clinical variants, the disease activity and the presence of comorbidities.

\section{Methods}

Cross-sectional observational study that included 184 patients with PsA according to CASPAR criteria. IL6 and TNF-a levels were determined. As clinical variables, the clinical form (peripheral, axial or mixed), the presence of dactylitis, the severity of psoriasis measured by PASI and HLA-B27 were determined. Disease activity was measured by the tender joint count, swollen joint count, entheses affected, the severity of psoriasis measured by PASI, ESR, and CRP. The minimum disease activity (MDA) was also measured. Cardiovascular risk markers such as waist / hip ratio $(\mathrm{w} / \mathrm{h})$ and analytical variables: apolipoprotein $\mathrm{A}$, apolipoprotein B, lipoprotein a, insulin, insulin resistance (HOMA-R) and microalbuminuria in urine 24 hours (MA) were included in comorbidity. The presence of fatty liver was measured by ultrasound and fatigue by the FACIT-F questionnaire.

\section{Results}

The mean age of the patients was 55.12 years (SD: 11.29). One hundred and one were men (54.89\%). $14.67 \%$ of the patients were on treatment with biologic DMARD (bDMARD). One hundred and two patients had peripheral involvement (55.43\%), 69 mixed (37.5\%) and $13(7.07 \%)$ exclusively axial. $17.93 \%$ of the patients had a positive HLA-B27. 53.26\% of the patients achieved a MDA. In the analysis of IL-6, we found a correlation with CRP (R: 0.32; $P=0.001)$, in addition, in patients with positive HLA-B27 we found lower concentrations of IL-6 $(3.25+2,26$ Vs $5.81+7.23)-p<0.001)$. We found no association with other variables related to inflammatory activity and / or comorbidity. TNF-a concentrations were higher in patients receiving TNF-a inhibitors ((178.89 SD: 181.31 vs 10.42 SD: 11.15; $P<0.001)$. Excluding these patients, we only found a correlation with the MA $(R: 0.39 ; p<0.001)$.

\section{Conclusions}

In our patients, the presence of HLA-B27 influenced IL- 6 concentrations. TNF-a could be considered as a marker of subclinical renal damage.

\section{Introduction}

Psoriatic arthritis (PsA) is a chronic inflammatory musculoskeletal disease that can affect the peripheral joints, the spine, or the enthesis. Activation of the Th1 and Th17 lymphocyte-mediated response is 
associated with the onset and perpetuation of the disease. IL- 6 is a pro-inflammatory cytokine that modulates the immune response. In patients with PsA, IL- 6 together with IL-1 $\beta$ induces the transcription of IL-23R and IL-23 and the differentiation of IL-17, this alternative pathway, not subject to self-regulation seems to influence the pathogenesis of the Disease [1]. From a clinical point of view, higher serum concentrations of IL- 6 have been found in patients with PSA compared to patients with ostearthritis and healthy subjects [2]. Furthermore, levels of IL-6 could influence the disease activity measured by the Swollem Joint Count (SJC), ESR, and CRP [3]. Similarly, the decrease in IL-6 levels in synovial fluid, after treatment with a TNF-a inhibitor, was associated with a Clinical improvement [4]. On the other hand, the polymorphism of IL-6 $(-174 \mathrm{G}>\mathrm{C})$ has been related to the peripheral forms of PsA, so that IL-6 could be related to the clinical form of the disease [5]. Although the role of IL-6 in the comorbidity of other diseases is known, there is not much information in this regard in patients with PsA [6, 7].

Th17 cells stimulate TNF-a production with the known effects on inflammation, cell matrix degradation, and bone resorption. In patients with PsA, serum TNF-a concentrations are higher than those found in patients with psoriasis and healthy subjects [8]. Higher concentrations of TNF-a have also been found in the synovial fluid of patients with PsA compared to patients with osteoarthritis [9]. Although TNF-a inhibition reduces inflammatory activity in patients with PsA, a recent study found no relationship between serum TNF-a levels and disease activity [10]. From a metabolic point of view, TNF-a promotes insulin resistance, increases plasma triglycerides and is associated with weight gain [11].

The objective of our work was to relate IL- 6 and TNF-a concentrations with the clinical form, the presence of dactylitis, the disease activity and comorbidity.

\section{Methods}

\section{Study design and population}

Cross-sectional observational study in 184 patients with PsA diagnosed by the CASPAR criteria who attended a tertiary hospital consultation consecutively [12]. Due to the possible interaction with the measured variables associated with cardiovascular comorbidity, patients diagnosed with high blood pressure and / or diabetes mellitus were excluded.

\section{Data collection and clinical measures}

IL-6 and TNF-a levels (IMMULITE and IMMULITE 1000 systems. Solid-phase, chemiluminescent immunometric assay. Siemens Healthcare Diagnostics Products Ltd. Glyn Rhonwy, Llanberis, Gwynedd LL55 4EL United Kingdom) were determined.

As clinical variables, age, gender, clinical form (peripheral, axial or mixed), dactylitis and HLA-B27 (PCRSSOr luminex) were measured. Axial disease was defined as inflammatory low back pain along with bilateral grade II or unilateral grade III sacroiliitis and / or syndesmophytes. As disease activity, the tender joint count (TJC), swollen joint count (SJC), the visual analogue pain scale (VAS), the number of painful 
entheses (measured by the MASES index [13]), the PASI [14], ESR and CRP. The therapeutic target was measured by the minimal disease activity [15]. The following variables were measured in comorbidity: waist / hip ratio ( $w / h)$, apolipoprotein $A$, apolipoprotein $B$, lipoprotein a, insulin, insulin resistance ( HOMA-R), 24-hour urine microalbuminuria (MA), the presence of fatty liver (measured by ultrasound) and fatigue (FACIT-F questionnaire [16]). Due to the possible influence of serum TNF-a levels in patients treated with TNF-a inhibitors, the statistical analysis was performed with the total number of patients and excluding patients treated with TNF-a inhibitors.

\section{Statistical Analysis}

The statistical analysis was performed using SPSS version 20. Variables were examined by testing frequency dispersion and normality criteria. The influence of each variable was measured in univariate regression tests. Finally, the variables that were significant in the univariate analysis were weighted using logistic regression or conditional forward stepwise (forward step) methods. In the bivariate correlations, the Pearson correlation coefficient was used $(p<0.05)$.. The study was approved by the ethics committee of the Hospital Clínico Universitario de Salamanca (EO 19/123).

\section{Results}

\section{A) General characteristics of the population}

The mean age of the patients was 55.12 years (SD: 11.29). One hundred and one were men $(54.89 \%)$. Eighty-nine were on methotrexate treatment (48.36\%), 44 with salazopyrine $(23.91 \%)$ and 18 with leflunomide (9.78\%). Twenty-seven patients (14.67\%) were on treatment with bDMARDs, 23 with TNF inhibitors, 2 with Ustekinumab and another 2 with Secukinumab. One hundred and two patients had peripheral involvement (55.43\%), 69 mixed (37.5\%) and 13 (7.07\%) exclusively axial. $17.93 \%$ of the patients had a positive HLA-B27. 53.26\% of the patients achieved a MDA. The baseline characteristics of the population are shown in Table 1. 
Table 1

Baseline characteristics

\begin{tabular}{|ll|}
\hline Age (years) & $\mathbf{5 5 , 1 2}(\mathbf{1 1}, 29)$ \\
\hline Disease duration (years) & $11,21(8,29)$ \\
\hline Gender (n/\%) & $101(54,89)$ \\
Male & $83(45,11)$ \\
Female & \\
\hline Clinical form (n/\%) & $102(55,43)$ \\
Peripheral & $69(37,50)$ \\
\hline Mixed & $13(7,07)$ \\
\hline Axial & \\
\hline Dactilytis(n/\%) & $33(17,93)$ \\
\hline HLA-B27(n/\%) & $33(17,93)$ \\
\hline DMARD treatment (n/\%) & $151(82,06)$ \\
\hline Methotrexate & $89(48,36)$ \\
\hline Salazopyrine & $44(23,91)$ \\
\hline Leflunomide & $18(9,78)$ \\
\hline bDMARD treatment (n/\%) & $27(14,67)$ \\
\hline TNF inhibitors & $23(12,5)$ \\
\hline Secukinumab & $2(1,08)$ \\
\hline Ustekinumab & $2(1,08)$ \\
\hline MDA (n/\%) & $98(53,26)$ \\
\hline SJC & $1,62(1,10)$ \\
\hline TJC & $1,61(1,16)$ \\
\hline Number of enthesis & $1,27(1,10)$ \\
\hline PASI & $1,17(1,51)$ \\
\hline HAQ & $0,60(0,57)$ \\
\hline CRP (mg/dL) & $160,7(26,49)$ \\
\hline W/H ratio & \\
\hline Apolipoprotein A (mg/dL) & \\
\hline
\end{tabular}




\begin{tabular}{|ll|}
\hline Age (years) & $\mathbf{5 5 , 1 2}(\mathbf{1 1 , 2 9 )}$ \\
\hline Apolipoprotein B(mg/dL) & $95,83(27,01)$ \\
\hline Lipoprotein A(mg/dL) & $43,62(45,70)$ \\
\hline C Peptide $(\mathrm{ng} / \mathrm{mL})$ & $2,56(1,18)$ \\
\hline Insulin $(\mu \mathrm{U} / \mathrm{mL})$ & $20,00(93,58)$ \\
\hline HOMA & $2,73(2,49)$ \\
\hline Microalbuminuria $(\mathrm{mg} / \mathrm{L})$ & $15,54(11,36)$ \\
\hline Liver ultrasound & $70(38,04)$ \\
Esteatosis (n/\%) & $114(61,96)$ \\
Normal(n/\%) & \\
\hline TNF-a $(\mathrm{pg} / \mathrm{mL})$ & $40,03(132,41)$ \\
\hline FACIT-F & $36,50(11,09)$ \\
\hline IL-6 (pg/mL) & $5,41(4,67)$ \\
\hline
\end{tabular}

B) Relationship of IL-6 with clinical, activity and comorbidity variables.

We found a correlation with the CRP $(R: 0.32 ; P=0.001)$. We did not find a correlation with the number of affected entheses (R: 0.03; $P=0.7)$, NAT (R: $0.12 ; P=0.1)$, TJC (R: $0.10 ; P=0.1)$, VAS (R: $0.01 ; P=0.9$ ), PASI (R: 0.02; $P=0.7)$, ESR (R: $0.13 ; P=0.1), w /$ h ratio $(R: 0.1 ; P=0.1)$, apolipoprotein $A(R:-0.14 ; P=0.2)$, apolipoprotein $B(R:-0.05 ; P=0.5)$, lipoprotein a $(R:-0.03 ; P=0.7)$, insulin ( $R: 0.11 ; P=0.1)$, HOMA-R ( $R$ : $0.21 ; P=0.1)$, MA (R: $-0.02 ; P=0.8)$, FACIT-F ( $R:-0.03 ; P=0.7)$. The rest of the results are shown in Table 2.

Table 2

Relationship of IL-6 with qualitative variables

\begin{tabular}{|llc|}
\hline & Mean (SD) & p \\
\hline bDMARD (Yes/No) & $5,72(5,20) / 5,23(4,24)$ & 0,89 \\
\hline Dactilytis (Yes/No) & $7,32(4,61) / 5,30(4,67)$ & 0,24 \\
\hline MDA (Yes/No) & $4,96(4,25) / 6,00(6,18)$ & 0,30 \\
\hline Axial/mixed/peripheral & $4,68(4,20) / 5,97(5,73) / 5,27(4,38)$ & 0,49 \\
\hline HLA-B27 (+/-) & $3,25(2,26) / 5,81(7,23)$ & 0,001 \\
\hline Axial + Mixed + HLA-B27 (+)/Axial + Mixed + HLA-B27 (-) & $6,23(6,57) / 4,25(7,01)$ & 0,01 \\
\hline Esteatosis/normal & $6,07(4,79) / 4,25(3,54)$ & 0,16 \\
\hline
\end{tabular}


C) Relationship of TNF-a with clinical, activity and comorbidity variables.

We did not find a correlation between the number of affected entheses (R: 0.11; $P=0.2)$, SJC (R: 0.07; $P=$ $0.4)$, NAD ( $R: 0.1 ; P=0,09)$, VAS ( $R:-0.03 ; P=0.9)$, ESR (R: $0.13 ; P=0.1)$, PASI (R: $0.08 ; P=0.3)$, w / h ratio $(R: 0.10 ; P=0.2),:$ apolipoprotein $A(R:-0.05 ; P=0.6)$, apolipoprotein $B(R: 0.02 ; P=0.7)$, lipoprotein a (R: $-0.11 ; P=0.2)$, insulin ( $R:-0.02 ; P=0.8)$, HOMA-R ( $:-0.13 ; P=0.4)$, MA (R: $0.3 ; P=0.001)$ and FACIT-F (R: $0.13 ; P=0.1)$. The rest of the results are set forth in Table 3 .

Table 3

Relationship of TNF-a with qualitative variables

\begin{tabular}{|lll|}
\hline & Mean (SD) & p \\
\hline bDMARD(Yes/No) & $180,68(176,20) / 12,57(10,12)$ & 0,001 \\
\hline Dactilytis(Yes/No) & $24,75(18,75) / 57,48(37,95)$ & 0,29 \\
\hline MDA (Yes/No) & $74,86(62,66) / 18,93(9,20)$ & 0,009 \\
\hline Axial/mixed/peripheral & $50,70(37,32) / 63,12(97,98) / 28,86(18,79)$ & 0,19 \\
\hline HLA-B27 (+/-) & $72,68(67,24) / 45,58(41,42)$ & 0,23 \\
\hline Esteatosis/normal & $46,02(40,83) / 38,61(3,27)$ & 0,70 \\
\hline
\end{tabular}

Serum TNF-a concentrations were different in patients treated with iTNF- $a$ and those not treated (178.89 SD: 181.31 vs 10.42 SD: $11.15 ; P<0.001)$. Due to the influence of treatment with TNF-a inhibitors on TNFa concentrations, we also performed the analysis excluding patients treated with TNF-a inhibitors, finding a correlation only with MA (R: $0.39 ; p<0.001)$. Regardless of the treatment with TNF-a inhibitors, we found no correlation between MA and NSAID intake $(p=0.3)$.

\section{Discussion}

Leukocyte antigen HLA-B27 influenced serum IL-6 concentrations. From a clinical point of view, in the axial forms (pure or mixed) not associated with HLA-B27, IL-6 concentrations were higher than in these clinical forms where the HLA-B27 was present. Serum levels of IL-6 and TNF-a had no impact on the inflammatory activity of the disease measured by joint swelling, by the number of entheses affected or by the severity of psoriasis. Neither did they influence fatigue or variables related to cardiovascular comorbidity, except for the association of serum TNF-a levels with subclinical renal damage.

Regarding the association between serum levels of IL- 6 and the presence of HLA-B27, indirectly, these results agree with a previous study, where the relationship between the IL-6 polymorphism (-174 G / C) and the presence of HLA-B27, in it, the $G$ allele of the polymorphism, related to an increase in transcriptional activity and secondarily with higher levels of IL-6, was significantly associated with negative HLA-B27 $[5,17]$. 
Although we found a strong correlation between IL- 6 concentrations and CRP, (related to hepatic release of IL-6-induced acute phase reactants) we found no relationship with other clinical parameters of inflammation [18]. In a Swedish study, IL-6 levels were related to SJC, using the Moll and Wright criteria, this study obtained higher concentrations of IL- 6 among patients with joint involvement associated with rheumatoid factor [19].

However, the increased expression of IL-6 in the synovium of patients with PsA could have demonstrated that IL- 6 inhibition could be useful in the treatment of PsA. At the moment the results have not been categorical, in most of the patients treated with Tocilizumab, the inhibition of IL- 6 was correlated with a decrease in of CRP levels, although a decrease in the clinical activity of the patients did not always occur $[20,21]$. Recently, in a randomized study, IL-6 blockade with clazakizumab showed efficacy in joint manifestations, measured by both the ACR20 response (at the $100 \mathrm{mg}$ dose; 52.4 vs $29.3 ; p<0.039$ ) as by SJC and / or TJC. There was also a response in enthesitis and dactylitis. There was no improvement in skin parameters [22]. In our study, as in other studies, there was no relationship between IL-6 levels and skin involvement measured by PASI [8].

Neither did we find a correlation between the serum TNF a level and SJC, TJC, VAS, enthesitis, dactylitis, CRP or ESR. In a recent study, Caso et al. found similar results [10]. In patients with spondylitis, there was also no relationship between serum TNF-a and ESR or CRP [23]. In our study, serum TNF a concentrations were higher in patients who were receiving TNF a inhibitors. This finding may be due to the fact that TNFa blockade causes secondary hyperproduction of TNF-a. Furthermore, patients achieving a MDA had higher concentrations of TNF-a. This result would be secondary to the higher proportion of treatment with TNF-a inhibitors in the group of patients who achieved a MDA. Excluding patients who were treated with TNF-a inhibitors, we also found no correlation between TNF-a and activity parameters or PASI. In previous studies, although patients with psoriasis had higher concentrations of TNF- $a$ than in controls, these also did not correlate with the severity of psoriasis [8].

In our study, the intensity of fatigue was not correlated with the concentrations of IL- 6 or TNF- $a$. The multifactorial etiology of fatigue may justify this result. Indeed, although it has been documented in animal models that IL- 6 concentrations are related to muscle weakness and that blocking IL- 6 and TNF a in patients with PsA produces a slight improvement in fatigue. However, the intensity of fatigue may be due to other causes, such as emotional disturbances, not directly linked to inflammation [22, 24-27].

Adipose tissue is metabolically active and produces IL-6 or TNF-a that can influence the inflammatory activity of the disease [28. On the other hand, the involvement of IL-6 in vascular morbidity associated with obesity is controversial, although previous studies it has been correlated with the presence of atherosclerosis in the coronary arteries. However, the administration of IL- 6 improves the use of insulin and the oxidation of fatty acids, in addition treatment with IL-6 inhibitors increases cholesterol concentrations [6, 29-31].

In our study, we did not find an association between serum IL-6 levels and the waist / hip ratio or the other parameters associated with obesity. These results coincide with those obtained with TNF a, except for the 
association with MA. MA is a marker of generalized vascular dysfunction that behaves as a cardiovascular risk factor [32]. Currently, there are no references to the presence of MA in patients with PsA, however, there are studies that link inflammation with MA. In patients with rheumatoid arthritis (RA), MA was more frequent than in healthy subjects, and it was also associated with inflammatory parameters such as DAS28, ESR, and ultrasensitive CRP [33]. In these patients, the severity of the MA was greater according to the degree of inflammation measured by the number of joints affected. In our study, however, there was no correlation with clinical disease activity, CRP, or ESR (Data not shown). In other studies with RA patients, there was no relationship between MA and TNF-a or IL-6 levels [34]. In patients with diabetes, the presence of soluble TNF- $a$ and IL- 6 receptors has been associated with MA. The TNF- $a$ / IL-6 pathway would be activated in up to $40 \%$ of patients with MA, with which these cytokines could be used as biomarkers of early kidney damage [35]. Inflammation can cause kidney damage through different mechanisms. TNF-a acts on Th1 lymphocytes, increasing the adhesion molecules of the renal vascular endothelium and promoting the migration of lymphocytes and leukocytes that cause endothelial dysfunction and associated kidney damage [36].

One of the limitations of the study is its cross-sectional nature, longitudinal studies would better catalog patients with sustained elevations of IL- 6 and TNF- $a$ and their consequences on activity, clinical form and comorbidities.

\section{Conclusions}

In summary,we can say that HLA-B27 can condition IL-6 levels and this is a potential explanation of why IL-6 inhibitors do not seem to offer benefits in B27 dependent diseases. On the other hand, TNF-a levels could be involved in early kidney damage. This finding is relevant because renal comorbidity has hardly been investigated in PsA. Therefore, it seems that apart from their role in pathogenesis, both cytokines play an essential role in the genesis of certain comorbidities (TNF-kidney) and may help explain the lack of response of certain drugs (anti-IL6) in HLA B27-dependent diseases.

\section{Abbreviations}

PsA

Psoriatic arthritis

MDA

Minimum disease activity

MA

Microalbuminuria in urine 24 hours

bDMARD

Biologic DMARD

HOMA-R

Insulin resistance

TJC 
Tender joint count

SJC

Swollen joint count

VAS

Visual analogue pain scale

\section{Declarations}

\section{Ethics approval and consent to participate}

The study was approved by the ethics committee of the Hospital Clínico Universitario de Salamanca (EO 19/123).

\section{Availability of data and materials}

The datasets used and/or analysed during the current study are available from the corresponding author on reasonable request.

\section{Competing interests}

All authors declare no conflicts of interest in this manuscript.

\section{Funding}

The source of support in the form of grants or industrial support: None.

\section{Authors'contributions}

CM substantially contributed to the acquisition of data, performed the statistical analysis, critically interpreted the results and wrote the first draft of the manuscript. LGL contributed to the acquisition of data, critically interpreted the results and revised the manuscript. ET, EA,OC,SP,CH and RQ critically interpreted the results and revised the manuscript. All authors read and approved the final version of the manuscript.

\section{References}

1. Ghoreschi K, Laurence A, Yang XP, Tato CM, McGeachy MJ, Konkel JE, Ramos HL, Wei L, Davidson TS, Bouladoux N, Grainger JR, Chen Q, Kanno Y, Watford WT, Sun HW, Eberl G, Shevach EM, Belkaid Y, Cua DJ, Chen W, O'Shea JJ. Generation of pathogenic $T(H) 17$ cells in the absence of TGF- $\beta$ Nature 2010: 467( 7318): 967-71

2. Chandran V, Abij F, Perruccio AV, Gandhi R, Li R, Cook RJ, Gladman DD Serum-based soluble markers differentiate psoriatic arthritis from osteoarthritis. Ann Rheum Dis 2019;78:796-801 
3. G.-M. Alenius, C. Eriksson2,S. Rantapää Dahlqvist1 Clinical and Experimental Rheumatology 2009 ;.Interleukin- 6 and soluble interleukin-2 receptor alpha-markers of inflammation in patients with psoriatic arthritis? Clin Exp Rheumatol. 2009 Jan-Feb;27(1):120-3.

4. Flocco U, Sfriso P, Roux-Lombard P, Scagliori E, Cozzi L Lunardi F et al.Synovial effusion and synovial fluid biomarkers in psoriatic arthritis to assess intraarticular tumor necrosis factor-a blockade in the knee joint. Arthritis Res Ther 2010; 12(4): R148.

5. Cubino N, Montilla C, Usategui-martín R, Cieza-Borrela C, Carranco T, Calero-Paniagua I et al.Association of IL1B $(-511 \mathrm{~A} / \mathrm{C})$ and IL6 $(-174 \mathrm{G}>\mathrm{C})$ polymorphisms with higher disease activity and clinical pattern of psoriatic arthritis. Clin Rheumatol 2016; 35:1789-1794

6. z)Lee YH, Pratley RE. The evolving role of inflammation in obesity and the metabolic síndrome. Curr Diab Rep 2005; 5:70-5.

7. Meyers CA, Albitar M, Estey E. Cognitive impairment, fatigue, and cytokine levels in patients with acute myelogenous leukemia or myelodysplastic syndrome. Cancer 2005; 104: 788-793.

8. Ozer Arican, Murat Aral, Sezai Sasmaz, Pinar Ciragil Serum levels of TNF-alpha, IFN-gamma, IL-6, IL8, IL-12, IL-17, and IL-18 in patients with active psoriasis and correlation with disease severity. Mediators of Inflammation 2005; 5:273-279

9. Partsch $G^{1}$, Steiner G, Leeb BF, Dunky A, Bröll H, Smolen JS Highly increased levels of tumor necrosis factor-alpha and other proinflammatory cytokines in psoriatic arthritis synovial fluid. $J$ Rheumatol.1997;24(3):518-23.

10. Caso F, Postiglione L, Covelli B, Ricciardone M, Di Spigna G, Formisano P, D'Esposito V, Girolimetto N, Tasso M, Peluso R, Navarini L, Ciccozzi M, Margiotta DPE, Oliviero F, Afeltra A, Punzi L, Del Puente A, Scarpa R, Costa L. Pro-inflammatory adipokine profile in psoriatic arthritis: results from a crosssectional study comparing PsA subset with evident cutaneous involvement and subset "sine psoriasis".Clin Rheumatol.2019 May 30. doi: 10.1007/s10067-019-04619-w

11. Sethi JK, Hotamisligil GS (1999) The role of TNF alpha in adipocyte Semin Cell Dev Biol 10:19-29

12. Taylor W, Gladman D, Helliwell P, Marchesoni A, Mease P, Mielants $H$, and the CASPAR Study Group Classification Criteria for Psoriatic Arthritis Development of New Criteria From a Large International Study Arthritis Rheumatol 2006; 54(8):2665-2673.

13. Heuft-Dorenbosch L, Spoorenberg A,van Tubergen A, Landewe R, van der Tempel $H$, Mielants $H$, et al. Assessment of enthesitis in ankylosing spondylitis. Ann Rheum Dis 2003; 62: 127-32

14. Fredriksson T, Pettersson U. Severe psoriasis: oral therapy with a new retinoid. Dermatologica 1978; 157: 238-44

15. Coates LC, Fransen J, Helliwell PS. Defining minimal disease activity in psoriatic arthritis: a proposed objective target for treatment. Ann Rheum Dis 2010;69(1):48-53

16. Cella D, Yount S, Sorrensen M, et al. Validation of the Functional Assessment of Chronic Illness Therapy Fatigue Scale relative to other instrumentation in patients with rheumatoid arthritis. $J$ Rheumatol 2005; 32: 811-819. 
17. Fishman D, Faulds G, Jeffery R, Mohamed-Ali V, Yudkin JS, Humphries S, Woo P. The effect of novel polymorphisms in the interleukin-6 (IL-6) gene on IL-6 transcription and plasma IL-6 levels, and an association with systemic-onset juvenile chronic arthritis. J Clin Invest. 1998;102:1369-76.

18. Yudkin JS, Stehouwer CD, Emeis JJ, Coppack SW.C-reactive protein in healthy subjects: associations with obesity, insulin resistance, and endothelial dysfunction: a potential role for cytokines originating from adipose tissue? Arterioscler Thromb Vasc Biol 1999;19:972-8

19. A W R van Kuijk, P Reinders-Blankert, T J M Smeets, B A C Dijkmans, P P Tak. Detailed analysis of the cell infiltrate and the expression of mediators of synovial inflammation and joint destruction in the synovium of patients with psoriatic arthritis: implications for treatment. Ann Rheum Dis 2006;65:1551-1557.

20. Ogataa A, Umegakib N, Katayamab I, Kumanogoha A, Tanakaa T. Psoriatic arthritis in two patients with an inadequate response to treatment with tocilizumab. Joint Bone Spine 2012; 79: 85-87.

21. Hughes $M$, Chinoy $H$. Successful use of tocilizumab in a patient with psoriatic arthritis. Rheumatology 2013;52:1728-1729

22. Mease PJ, Gottlieb AB, Berman A, Drescher E, Xing J, Wong R, Banerjee S. The Efficacy and Safety of Clazakizumab, an Anti-Interleukin-6 Monoclonal Antibody, in a Phase Ilb Study of Adults With Active Psoriatic Arthritis. Arthritis Rheumatol.2016; 68:2163-73.

23. Gonzalez-López L, Fajardo-Robledo NS, Saldaña-Cruz A, Moreno-Sandoval IV, Bonilla-Lara D, Zabaleta-Muñiz et al. Association of adipokines, interleukin-6, and tumor necrosis factor-a concentrations with clinical characteristics and presence of spinal syndesmophytes in patients with ankylosing spondylitis: A cross-sectional study. J Int Med Res.2017;45:1024-1035.

24. Hart BL. Biological basis of the behavior of sick animals. Neurosci. Biobehav Rev 1988; 12:123-37

25. Reich K, Segaert S, Van de Kerkhof P, et al. Once-weekly administration of etanercept 50 mg improves patient-reported outcomes in patients with moderate-to-severe plaque psoriasis.Dermatology 2009; 219: 239-249.

26. Lebwohl M, Papp K, Han C, et al. Ustekinumab improves health-related quality of life in patients with moderate-to-severe psoriasis: results from the PHOENIX 1 trial. Br J Dermatol 2010; 162: 137-146.

27. Papp K, Crowley J, Ortonne JP, et al. Adalimumab for moderate to severe chronic plaque psoriasis: efficacy and safety of retreatment and disease recurrence following withdrawal from therapy. $\mathrm{Br} \mathrm{J}$ Dermatol 2011; 164: 434-441

28. Aprahamian TR, Sam F. Adiponectin in cardiovascular inflammation and obesity. Int J Inflam 2011;2011:3769

29. Kimura A, Naka T, Kishimoto T. IL-6-dependent and -independent pathways in the development of interleukin 17-producing T helper cells. Proc Natl Acad Sci USA. 2007;104:12099-104.

30. Yu J, Xiao Z, Zhao R, et al. Astilbin emulsion improves guinea pig lesions in a psoriasis-like model by suppressing IL-6 and IL-22 via p38 MAPK. Mol Med Rep. 2018;17:3789-96

31. Double-blind randomized controlled clinical trial of the interleukin-6 receptor antagonist, tocilizumab, in European patients with rheumatoid arthritis who had an incomplete response to methotrexate. 
Maini RN, Taylor PC, Szechinski J, Pavelka K, Bröll J, Balint G, Emery P, Raemen F, Petersen J, Smolen J, Thomson D, Kishimoto T; CHARISMA Study Group. Arthritis Rheum. 2006 Sep;54:2817-29

32. Tsioufis C, Dimitriadis K, Antoniadis D, Stefanadis Ch, Kallikazaros I. Inter-relationships of microalbuminuria with the other surrogates of the atherosclerotic cardiovascular disease in hypertensive subjects. Am J Hypertens. 2004;17:470-6.

33. Nada DW, El Morsy S, Abu-Zaid MH, Aboelhawa MA, Zakaria MA, El Sheikh EA, Gaber RA. The role of microalbuminuria as a predictor of subclinical cardiovascular events in rheumatoid arthritis patients and its relation to disease activity. Clin Rheumatol 2018; 37:623-630

34. Becetti K, Omseth MJ, Solus JF, RaggiP, Stein CM, Chung CP. Urinary albumin excretion is increased in patients with rheumatoid arthritis and associated with arterial stiffness. J Rheumatol.2015;42:5938

35. Purohit S, Sharma A, Zhi W, Bai S, Hopkins D, Steed L et al. Proteins of TNF-a and IL6 Pathways Are Elevated in Serum of Type-1 Diabetes Patients with Microalbuminuria. Immunol., 31 January 2018

36. Wu CC, Sytwu HK, Lu KC, Lin YF. Role of T cells in type diabetic nephropathy Exp Diabetes Res. 2011;2011:514738. 